\title{
Angiotensin type 1 receptor regulates yes-associated protein in vascular endothelial cells
}

\author{
XINHAO WANG, HONGPENG ZHANG, YANGYANG GE, JIE LIU, DAN RONG, \\ LONG CAO, YUAN HE, GUOYI SUN, SENHAO JIA and WEI GUO \\ Department of Vascular and Endovascular Surgery, First Medical Center of \\ Chinese People's Liberation Army General Hospital, Beijing 100853, P.R. China
}

Received May 23, 2019; Accepted October 28, 2019

DOI: $10.3892 /$ etm.2019.8259

\begin{abstract}
Aortic dissection (AD) is one of the most lethal cardiovascular diseases. Endothelial cell (EC) dysfunction serves an important role in AD progression. Angiotensin II (Ang II) is a key effector in cardiovascular disease development that acts through binding to angiotensin type 1 receptor (AT1R). Yes-associated protein (YAP) is well-known as a key mediator of cell proliferation and apoptosis. To determine whether AT1R and YAP influence EC proliferation or injury, human aortic endothelial cells were cultured under different culture conditions. Using CCK- 8 assay, ELISA, western blotting, immunocytochemistry and siRNA transfection, the present study found that Ang II activity reduced EC proliferation, upregulate YAP phosphorylation and result in EC injury that was associated with elevated levels of multiple proinflammatory chemokines. The inhibition of AT1R function, pharmaceutically or via transfection with an AT1R small interfering RNA, alleviated the effects induced by Ang II. Furthermore, AT1R induced YAP phosphorylation via binding to Ang II, and further promoted the inflammation of ECs, along with inhibiting their proliferation.
\end{abstract}

\section{Introduction}

Aortic dissection (AD) is a fatal disease that accounts for a large proportion of aortic-associated mortalities (1). Indeed,

Correspondence to: Dr Wei Guo, Department of Vascular and Endovascular Surgery, First Medical Center of Chinese People's Liberation Army General Hospital, 28 Fuxing Road, Beijing 100853, P.R. China

E-mail: guoweiplagh@sina.com

Abbreviations: AD, aortic dissection; Ang II, angiotensin II; AT1R, angiotensin type 1 receptor; YAP, yes-associated protein; EC, endothelial cell; ARB, angiotensin receptor blocker; HAEC, human aortic endothelial cell; CCK-8, Cell Counting Kit-8; ET-1, endothelin-1; IL-6, interleukin-6; MMP9, matrix metalloproteinase 9

Key words: endothelial cell, yes-associated protein, angiotensin II, proliferation, angiotensin type 1 receptor epidemiological surveys have demonstrated that the incidence of thoracic AD is 3-4 per 100,000 individuals per year as of $2011(2,3)$. AD is defined as blood flow that enters the aortic media through intimal tears, followed by a formation of a false lumen addition to the true lumen (4). The false lumen wall is composed of the intima and media of the aortic wall, and the true lumen is the original aortic lumen. Clinically, AD can result in numerous serious complications, including aortic rupture and visceral ischemia (4). The majority of patients with non-operative ascending AD (Stanford type A) exhibit an overall survival time of $<2$ weeks (5). Unfortunately, due to its sudden and unpredictable nature, little is known regarding the pathological and molecular events underpinning the development or progression of AD.

Endothelial dysfunction serves an initial role in the development and pathogenesis of cardiovascular diseases, including AD (6). Vascular endothelial cells (VECs) are flat endothelial cells (ECs) in the inner lining of the major blood vessels. They are important in regulating blood flow, and thus, are involved in numerous physiological processes (6). Inflammation can result in VEC dysfunction and further promote AD progression (7). Angiotensin II (Ang II) is the major effector peptide of the renin-angiotensin system (RAS), which induces vasoconstriction, hypertrophy and extracellular remodeling via the angiotensin type 1 receptor (AT1R) (8). Ang II-induced endothelial dysfunction has been implicated in a variety of cardiovascular diseases, including atherosclerosis, hypertension, left ventricular hypertrophy, myocardial infarction and heart failure (9). Additionally, studies have indicated that Ang II contributes to the development of AD in humans and animal models (10), and also promotes the production of reactive oxygen species by inducing multiple downstream pathways in VECs (11). Angiotensin receptor blocker (ARB) is an antihypertensive drug that is commonly used to treat patients with AD by achieving blood pressure control (4). In the present study, the effect of $\mathrm{ARB}$ on $\mathrm{AD}$ progression was investigated. Both VEC dysfunction and Ang II serve an important role in the occurrence of $\mathrm{AD}$ and consequently the association between them was also investigated.

Yes-associated protein (YAP) is a pluripotent intracellular junction protein that is both a transcriptional co-activator, and a major effector of the Hippo-YAP signaling pathway. Nuclear YAP binds to the transcriptional enhancer-associated 
domain transcription factors to regulate transcriptional processes, and ultimately influence cell proliferation and apoptosis (12). Mammalian sterile 20-like kinase-1 and -2 are the central mediators of the Hippo-YAP signaling pathway, which promotes YAP phosphorylation via signal transduction. Notably, phosphorylated YAP (p-YAP) cannot enter the nucleus, and remains in the cytoplasm where it cannot regulate transcription (13). Furthermore, it has been demonstrated that YAP serves an important role in the mediation of the proliferation, migration, apoptosis and phenotypic transition of ECs (14) and vascular smooth muscle cells (15). Additionally, a previous study found that the Hippo-YAP signaling pathway is regulated by Ang II signaling, and that its reactivation induces apoptosis and proliferation in podocytes (16). To the best of our knowledge, the influence of the YAP signaling pathway on EC proliferation and injury, and whether YAP expression in ECs is regulated by AT1R, is yet to be elucidated. Therefore, the purpose of the present study was to explore AT1R-mediated regulation of YAP by Ang II in ECs. Therefore, the present study aimed to identify the mechanisms underlying the YAP-mediated promotion of proliferation, and suppression of cell injury, in Ang II-treated HAECs. It was hypothesized that YAP is a key mediator of VEC Ang II-associated toxicity.

\section{Materials and methods}

Cell culture. Human monocytic HAECs (Shanghai Zhong Qiao Xin Zhou Biotechnology Co., Ltd.) were cultured in RPMI-1640 medium (Thermo Fisher Scientific, Inc.) supplemented with $10 \%$ fetal bovine serum (Gibco; Thermo Fisher Scientific, Inc.), 2 mM L-Glutamine and 1\% penicillin, streptomycin and amphotericin-B (Sigma-Aldrich; Merck $\mathrm{KGaA}$ ) at $37^{\circ} \mathrm{C}$ and $5 \% \mathrm{CO}_{2}$. When the cells had adhered, they were transferred to phorbol myristate acetate-free medium (Sigma-Aldrich; Merck KGaA) to obtain resting HAECs. Cells (6x10 4 cells/well) were then seeded into multi-well plates with a membrane insert of $0.4-\mu \mathrm{m}$ pore size (Corning Life Sciences). Different factors were added to the wells after $24 \mathrm{~h}$ incubation as follows: Control group, HAECs only; group 1, HAECs with Ang II $(1 \mu \mathrm{M})$ treatment; group 2, HAECs with Ang II $(1 \mu \mathrm{M})$ and small interfering RNA (siRNA) negative control (NC) transfection; group 3, HAECs with Ang II $(1 \mu \mathrm{M})$ and AT1R siRNA transfection; group 4, HAECs with Ang II $(1 \mu \mathrm{M})$ and $1 \mu 1 \mathrm{DMSO}$; and group 5, HAECs with Ang II $(1 \mu \mathrm{M})$ and the ARB $1 \mu \mathrm{l}(20 \mathrm{mM})$ telmisartan treatment. After adding the various intervention conditions, the cells were further cultured for $72 \mathrm{~h}$ at $37^{\circ} \mathrm{C}$ and $5 \% \mathrm{CO}_{2}$. The total incubation time of the cells was $96 \mathrm{~h}$.

Cell Counting Kit (CCK)-8 assay. HAECs were inoculated into multi-well plates $\left(5 \times 10^{3}\right.$ cells/well). Cells were inoculated with three replicate sets per experimental group. Subsequently, CCK-8 was used according to the manufacturer's instructions. Briefly, CCK-8 solution (IS087, USCN Life Sciences, Inc.) was mixed with serum-free medium (1:10 v/v; DMEM with High Glucose; Gibco; Thermo Fisher Scientific, Inc.), and $10 \mu \mathrm{l}$ of the mixture was added to each well. Cells were then incubated at $37^{\circ} \mathrm{C}$ with $5 \% \mathrm{CO}_{2}$ for $4 \mathrm{~h}$, after which the optical density at $450 \mathrm{~nm}$ was determined.
ELISA. Endothelin (ET)-1 levels in cell culture supernatant from each treatment group at the end of the $96 \mathrm{~h}$ treatment period were detected using competitive inhibition ELISA with ET-1 Antibody ELISA kit (USCN Life Sciences, Inc.), and interleukin (IL)-6 and matrix metallopeptidase (MMP)-9 in the cell solutions were detected by double antibody ELISA with IL-6 ELISA Kit (USCN Life Sciences, Inc.) and MMP9 ELISA Kit (USCN Life Sciences, Inc.), respectively. ELISAs were performed in accordance with the manufacturer's protocols.

Western blotting for ATIR and YAP. Cell lysates were prepared using RIPA lysis buffer (P0013B, Beyotime Institute of Biotechnology) and total protein was extracted and quantified using a bicinchoninic acid protein concentration kit (cat. no. p0010; Beyotime Institute of Biotechnology) according to the manufacturer's protocol. Equal amounts of proteins $(20 \mu \mathrm{g})$ were separated via SDS-PAGE (15 g SDS, $15.6 \mathrm{ml}$ $2 \mathrm{M}$ Tris $\mathrm{pH}$ 6.8, $57.5 \mathrm{~g}$ glycerol, $16.6 \mathrm{ml} \beta$-mercaptoethanol) and transferred to PVDF membranes. The membranes were blocked with 5\% BSA (16000-044, Gibco; Thermo Fisher Scientific, Inc.) in Tris-buffered saline with $20 \%$ Tween-20 for $2 \mathrm{~h}$ at room temperature. Membranes were subsequently incubated overnight at $4^{\circ} \mathrm{C}$ with the following primary antibodies: Anti-YAP (cat. no. AF6328; polyclonal, rabbit anti-human and mouse; 1:1,000; Affinity Biosciences), anti-p-YAP (Ser127) (cat. no. AF3328; polyclonal, rabbit anti-human and mouse; 1:1,000; Affinity Biosciences), anti-AT1R (cat. no. DF4910; polyclonal, rabbit anti-human and mouse; 1:1,000; Affinity Biosciences), anti-GAPDH (cat. no. ab9485; polyclonal, rabbit anti-human and mouse; 1:2,500; Abcam). The membranes were washed three times using PBS, after which anti-rabbit immunoglobulin G (IgG; H+L; cat. no. BA1054; polyclonal, goat anti-rabbit; 1:5,000; Boster Biological Technology) was added and the membranes were incubated at $37^{\circ} \mathrm{C}$ for $1 \mathrm{~h}$. Bands were detected using a Gel Doc systems (Bio-Rad Laboratories, Inc). The density of the bands were assessed by quantitative densitometric analysis using ImageJ (V1.8.0, National Institutes of Health).

AT1R siRNA transfection. AT1R siRNA was designed and synthesized by Shanghai GenePharma Co., Ltd. as follows: AT1R-165, GCAUUAAUGCCUCCAUUUATT; AT1R-880, GCUCAAGCCCUGUCAGAUATT; NC, AAUGUACUC ACUACGACUGCG. AT1R siRNAs were transfected into cells with Lipofectamine ${ }^{\circledR} 2000$ (Thermo Fisher Scientific, Waltham, MA USA) according to the manufacturer's protocol. HAECs in a logarithmic growth phase with good growth status were inoculated into a 6 -well plate at $5 \times 10^{5}$ cells/well, and cultured overnight at $37^{\circ} \mathrm{C}$ in a $5 \% \mathrm{CO}_{2}$ incubator. At $2 \mathrm{~h}$ prior to transfection, they were mixed with serum-free MEM $\alpha$ medium (Gibco; Thermo Fisher Scientific, Inc.).

Reverse transcription-quantitative PCR (RT-qPCR). After siRNA transfection for $48 \mathrm{~h}$, AT1R relative mRNA was collected from transfected HAECs as described in the previous section (AT1R siRNA transfection). RevertAid First Strand cDNA Synthesis kit (cat. no. K1622, Thermo Fisher Scientific, Inc.) were used according to the manufacturer's protocol in order to reverse transcribe $2 \mu \mathrm{g}$ RNA into first-strand cDNA. 
FastStart Universal SYBR Green Master (Rox; 04913914001, Hoffmann-La Roche, Ltd.) was then used to perform quantitative PCR as follows: $2 \mathrm{~min}$ at $50^{\circ} \mathrm{C}, 10 \mathrm{~min}$ at $95^{\circ} \mathrm{C}$, and then 40 cycles of $15 \mathrm{sec}$ at $95^{\circ} \mathrm{C}$ and $60 \mathrm{sec}$ at $60^{\circ} \mathrm{C}$. Amplification was performed on a PCR System 7300 (Applied Biosystems; Thermo Fisher Scientific, Inc.). The $2^{-\Delta \Delta C q}$ method was used to analyze the results (17). In addition, $\beta$-actin was used as an internal reference gene to account for unknown sources of variation. siRNA-165 was used for subsequent experiments following the effectiveness of AT1R siRNA which is exhibited in Fig. 1.

Immunocytochemistry to detect YAP localization. ECs after $96 \mathrm{~h}$ culture were fixed with 3\% formaldehyde solution for 10-25 min at room temperature after washing with PBS. Fixed cells were washed three times with PBS, then permeabilized with 1\% Triton X-100 (Beyotime Institute of Biotechnology), incubated for 5-10 min at room temperature, and rewashed three times with PBS. Each sample site and 3\% BSA blocking solution was sealed at room temperature for $30 \mathrm{~min}$. YAP antibody (AF6328, Affinity Biosciences) was diluted 1:500, and membranes were blocked overnight in a humid chamber at $4^{\circ} \mathrm{C}$ and washed three times with PBS. The fluorescent CY3 goat anti-rabbit IgG (rhodamine-labeled; cat. no. BA1036; Boster Biological Technology) and 4',6-diamidino-2-phenylindole were added to membranes (both diluted 1:500) for 30-60 min at room temperature in the dark, followed by washing three times with PBS. Slides were enclosed with fluorescence-quench mount and observed using a confocal laser scanning microscope (x1,000 magnification).

Statistical analysis. Statistical analysis was performed using SPSS software (v19.0; IBM Corp.) to evaluate differences among groups. Comparisons among $>2$ groups were performed using one-way ANOVA followed by Tukey's post-hoc test. Data are presented as mean \pm standard deviation, and $\mathrm{P}<0.05$ was considered to indicate a statistically significant difference. All experiments were repeated in triplicates.

\section{Results}

Knockdown of siRNA-165 is more efficient compared with siRNA-880. The knockdown efficiency of siRNA-165 and siRNA-880 was determined using quantitative PCR (Fig. 1). The results indicated that siRNA-165 knockdown efficiency was more stable and the effect was more notable, and it was consequently selected for subsequent experimentation.

Ang II reduces VEC proliferation and triggers an inflammatory response, which is suppressed by treatment with $A R B$ and ATIR siRNA. Cell proliferation was investigated using a CCK-8 assay (Fig. 2A). Compared with the control group, cell proliferation was significantly decreased following treatment with Ang II ( $1 \mu \mathrm{M} ; 24 \mathrm{~h} ; \mathrm{P}<0.05)$. Additionally, compared with groups 1 and 2 (Ang II treatment with or without NC siRNA), cell proliferation was significantly increased following transfection with AT1R siRNA after culturing for $48 \mathrm{~h}$ (group 3; $\mathrm{P}<0.05$ ), and also increased following treatment with telmisartan after culturing for $48 \mathrm{~h}$ (group 4; $\mathrm{P}<0.05$ ). This meant that the inhibition of the proliferative activity of ECs by Ang II

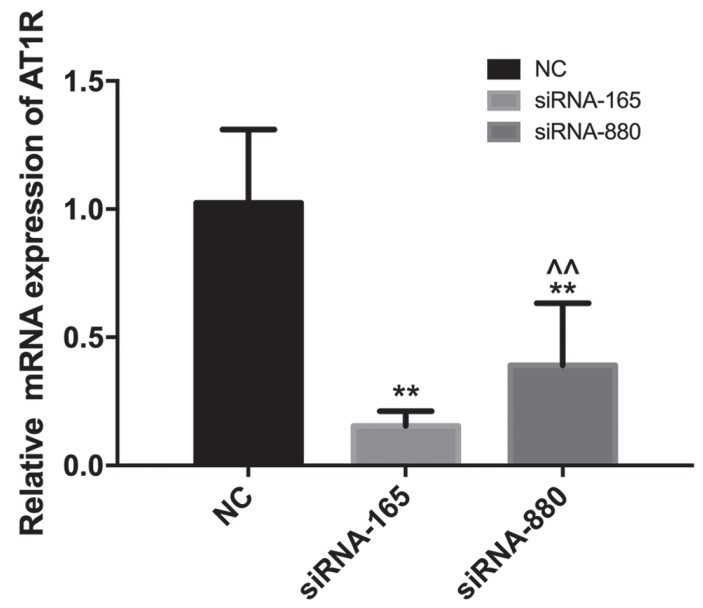

Figure 1. AT1R siRNA-165 exhibits better knockdown efficiency compared with siRNA-880. The knockdown efficiency of AT1R siRNA was detected by quantitative PCR. Mean $\pm \mathrm{SD} ;{ }^{* *} \mathrm{P}<0.05$ vs. siRNA NC; and ${ }^{\wedge \wedge} \mathrm{P}<0.05$ vs. siRNA-165. siRNA, small interfering RNA; NC, negative control; AT1R, angiotensin type 1 receptor.

is achieved by regulating AT1R. Based on the present results, it was speculated that Ang II induced an inflammatory reaction in HAECs. Since Ang II is known to upregulate ET-1, proinflammatory chemokines, such as interleukin-6 (IL-6), and MMP-9 to promote endothelial inflammation $(18,19)$, the expression levels of these factors were measured in supernatants from each group (Fig. 2B-D). Circulating levels of IL-6 and ET-1 were all significantly higher in groups 1,2 and 4 than in the control group and groups 3 and $5(\mathrm{P}<0.05)$. Group 5 and group 3 had a decrease in MMP 9 circulating levels compared with group 4 and group 2, respectively. The current findings indicated that Ang II promoted endothelial inflammation and that transfection with ARB and AT1R siRNA may alleviate these proinflammatory effects.

Ang II decreases the YAP/p-YAP ratio in VECs; however, treatment with $A R B$ reverses this effect. To identify alterations in YAP and AT1R expression in each group, western blotting was performed to detect AT1R, YAP and p-YAP expression in HAECs from each group (Fig. 3A). Compared with the control group, YAP expression was decreased in HAECs from groups 1,2 and 4 which were treated with Ang II (Fig. 3C), whereas AT1R (Fig. 3B) and p-YAP (Fig. 3D) expression was significantly increased in these groups $(\mathrm{P}<0.05)$. In groups 3 (transfection with AT1R siRNA) and 5 (treatment with telmisartan), these expression changes were alleviated to a certain extent (Fig. 3B-D). This indicated that Ang II promoted YAP phosphorylation, making it unable to enter the nucleus.

Ang II prevents YAP from entering the nucleus by binding to $A T 1 R$. To elucidate the intracellular localization of YAP, immunocytochemistry was performed for each treatment group and the control (Fig. 4A). Following incubation of HAECs for $72 \mathrm{~h}$ under different experimental conditions, levels of YAP in the nuclei of cells from groups 1,2 , and 4 were significantly reduced due to the influence of Ang II $(\mathrm{P}<0.05)$. This means that Ang II can prevent YAP from entering the nucleus. 

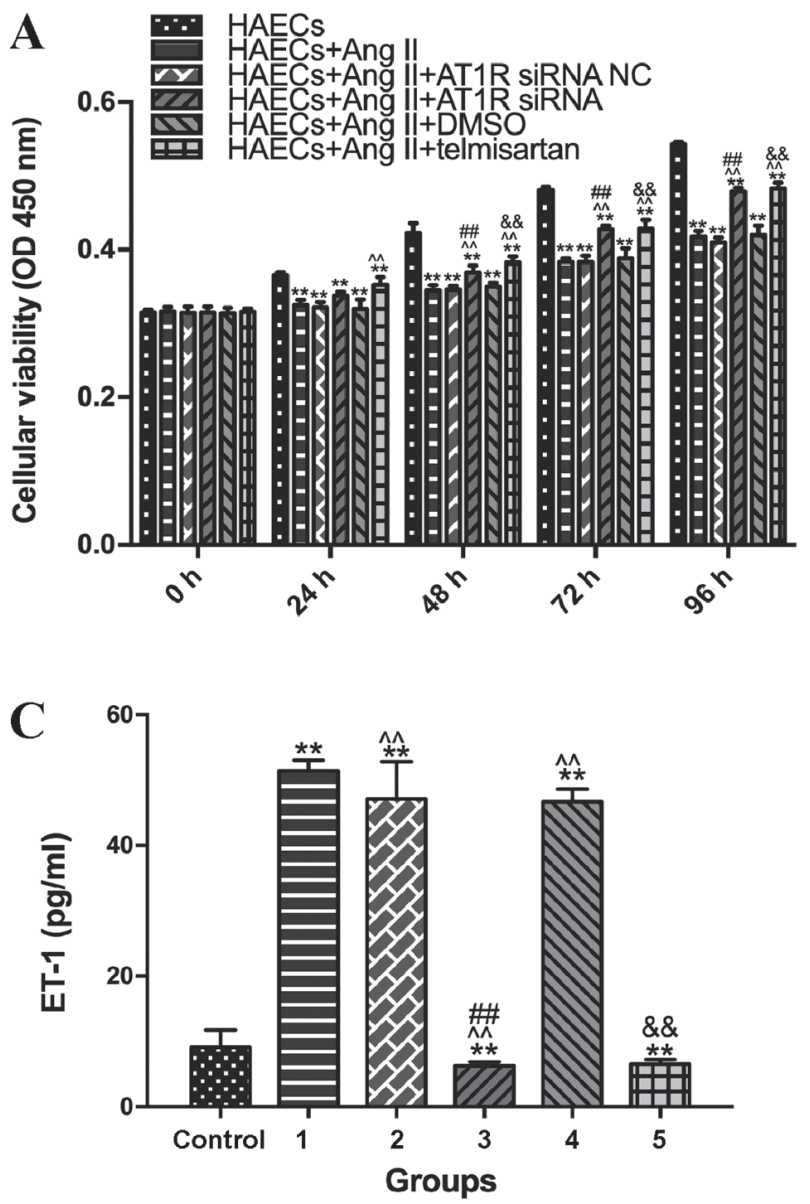

B
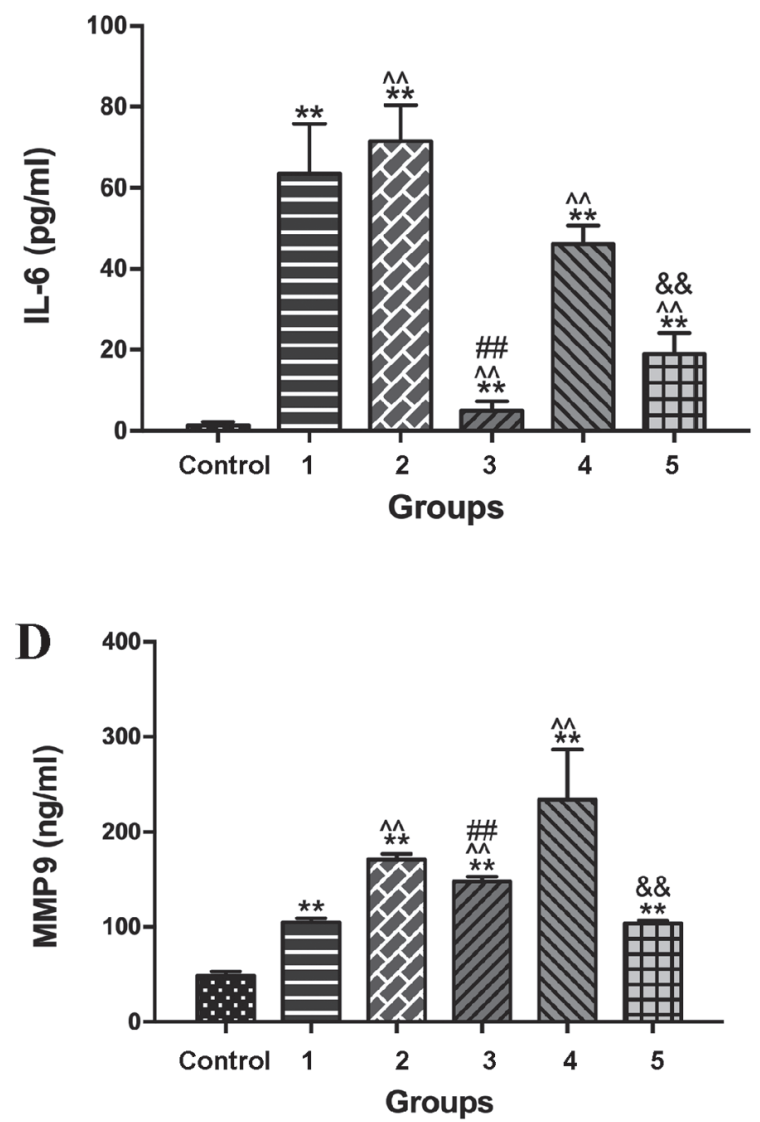

Figure 2. Ang II inhibits cell proliferation and promotes inflammation in HAECs, and telmisartan counteracts this effect. Grouping: Control group, HAECs only; group 1, HAECs with Ang II $(1 \mu \mathrm{M})$ treatment; group 2, HAECs with Ang II $(1 \mu \mathrm{M})$ and siRNA NC transfection; group 3, HAECs with Ang II (1 $\mu \mathrm{M})$ and AT1R siRNA transfection; group 4, HAECs with Ang II (1 $\mu \mathrm{M})$ and $1 \mu \mathrm{l}$ DMSO; and group 5, HAECs with Ang II (1 $\mu \mathrm{M})$ and the ARB $1 \mu \mathrm{l}(20 \mathrm{mM})$ telmisartan treatment. (A) CCK-8 detection of cell proliferation in HAECs at 0, 24, 48, 72 and $96 \mathrm{~h}$. After $96 \mathrm{~h}$, Ang II treatment $(1 \mu \mathrm{M})$ significantly decreased the proliferative activity of HAECs $(\mathrm{P}<0.05)$, and this decrease was significantly reduced by treatment with telmisartan (20 $\mu \mathrm{M})$. (B) Detection of IL-6 in supernatants from different samples by ELISA after $96 \mathrm{~h}$ culture. (C) Detection of ET-1 in supernatants from different samples by ELISA after $96 \mathrm{~h}$ culture. (D) Detection of MMP-9 in supernatants from different samples using ELISA after 96h culture. Ang II treatment $(1 \mu \mathrm{M})$ significantly increased the concentrations of IL-6, ET-1 and MMP9 in the supernatants compared with the control group (which was not treated with Ang II). IL-6, ET-1 and MMP-9 levels were significantly lower in the group 5 compared with the group $4 .{ }^{* *} \mathrm{P}<0.05$ compared with the group control; ${ }^{\wedge} \mathrm{P}<0.05$ compared with the group $1 ;$;" $\mathrm{P}<0.05$ compared with the group 4; \&\& $\mathrm{P}<0.05$ compared with the group 2. siRNA, small interfering RNA; NC, negative control; AT1R, angiotensin type 1 receptor; Ang II, angiotensin II; HAEC, human aortic endothelial cell; ET-1, endothelin-1; IL-6, interleukin-6; MMP9, matrix metalloproteinase 9.

Transfection of AT1R siRNA and treatment with telmisartan for $72 \mathrm{~h}$ effectively alleviated the cytoplasmic retention of YAP caused by Ang II (Fig. 4B and C; P<0.05). The current immunostaining data supported the results of western blotting, further indicating that Ang II promoted YAP phosphorylation via binding to AT1R, thereby preventing YAP from entering the nucleus.

\section{Discussion}

The present study demonstrated that Ang II binding to AT1R promoted YAP phosphorylation, upregulated YAP cytoplasmic retention and decreased HAEC proliferation. After quantifying ET1, MMP-9 and IL-6 expression levels, which are markers of inflammation and EC injury $(20,21)$, it was revealed that Ang II may trigger EC inflammation via upregulation of YAP phosphorylation. Furthermore, it was demonstrated that blocking AT1R reduced the Ang II-mediated inhibition of proliferation of VECs, downregulating YAP phosphorylation.
YAP is a well-characterized regulator of cell proliferation (12); hence, the present results demonstrated that Ang II binding with AT1R promoted YAP phosphorylation, which resulted in VEC inflammation and inhibited proliferation. This indicated that the AT1R-YAP signaling pathway serves an important role in Ang II-mediated intimal inflammation. However, the specific signaling pathway underlying this mechanism is yet to be elucidated.

Notably, the present study revealed that the aforementioned Ang II/AT1R-mediated YAP phosphorylation was inhibited following treatment with the specific AT1R inhibitor telmisartan or transfection with an AT1R siRNA, which initiated a change in YAP intracellular localization from the nucleus to the cytoplasm. G protein-coupled receptor binding to the $\mathrm{G}$ protein subclass of receptors $\left(\mathrm{G \alpha}_{\mathrm{q} / 11}\right)$ typically activates YAP (22) and AT1R activates YAP in HEK293 cells (16); hence, it was expected that AT1R may have the same effect in VECs. However, stimulation of AT1R with Ang II resulted in the upregulation of $\mathrm{p}$-YAP/YAP ratio in 
A
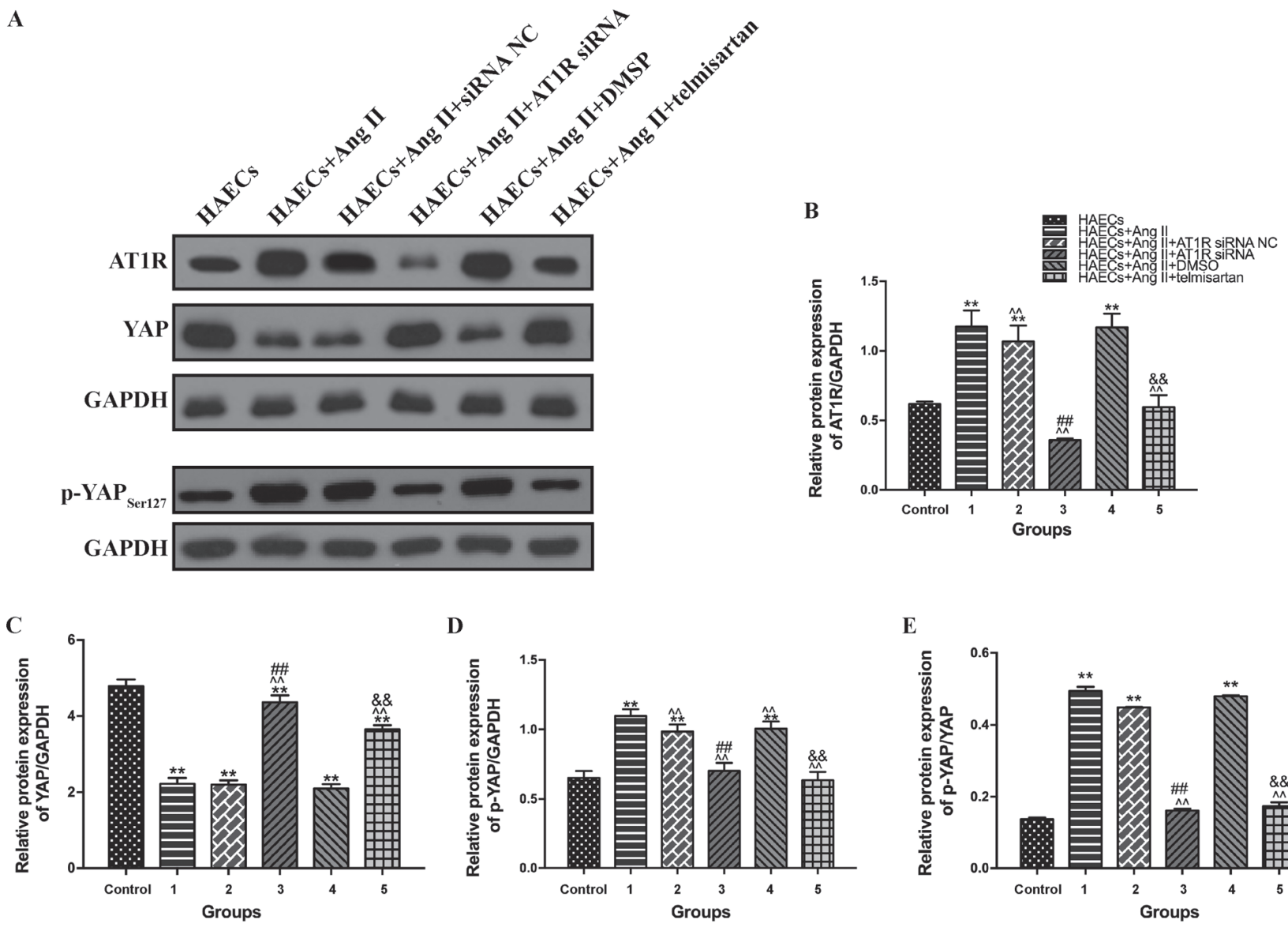

D

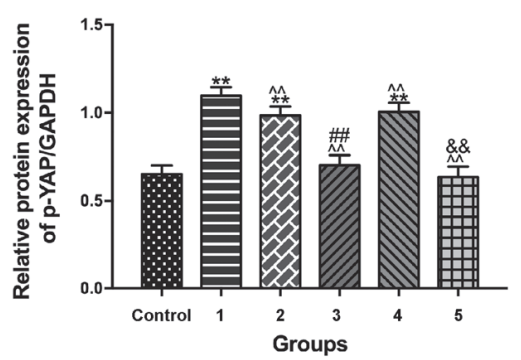

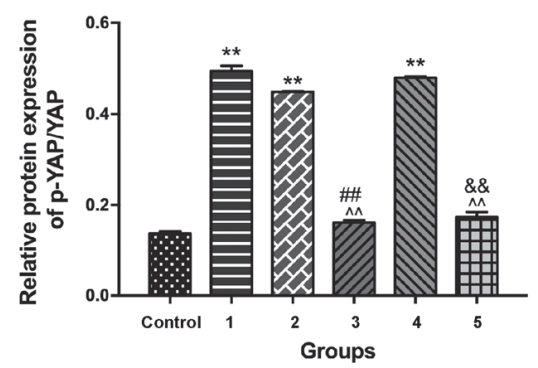

Figure 3. Ang II promotes AT1R expression and YAP phosphorylation (Ser127), and treatment with telmisartan or transfection with AT1R siRNA reverses this effect. Grouping: Control group, HAECs only; group 1, HAECs with Ang II (1 $\mu \mathrm{M})$ treatment; group 2, HAECs with Ang II (1 $\mu \mathrm{M})$ and siRNA NC transfection; group 3, HAECs with Ang II $(1 \mu \mathrm{M})$ and AT1R siRNA transfection; group 4, HAECs with Ang II (1 $\mu \mathrm{M})$ and $1 \mu \mathrm{l}$ DMSO; and group 5, HAECs with Ang II $(1 \mu \mathrm{M})$ and the ARB $1 \mu 1(20 \mathrm{mM})$ telmisartan treatment. (A) Western blot analysis of sets of six independent lysates from HAECs that were untreated, treated with Ang II, treated with Ang II and siRNA NC, treated with Ang II and AT1R siRNA, treated with Ang II and DMSO, or treated with Ang II and telmisartan. Treatment with Ang II upregulated AT1R and p-YAP, and this effect was alleviated by transfection with an AT1R siRNA or treatment with telmisartan. The ratio between (B) AT1R and GAPDH; (C) YAP and GAPDH; (D) p-YAP and GAPDH; and (E) p-YAP and YAP expression is presented. Mean $\pm \mathrm{SD}$. ${ }^{* *} \mathrm{P}<0.05$ vs. group control; ${ }^{\wedge} \mathrm{P}<0.05$ vs. group $1 ;{ }^{* \#} \mathrm{P}<0.05$ vs. group 4 ; ${ }^{\text {\&\&}} \mathrm{P}<0.05$ vs. HAECs + group 2 . siRNA, small interfering RNA; NC, negative control; AT1R, angiotensin type 1 receptor; Ang II, angiotensin II; HAEC, human aortic endothelial cell; ET-1, endothelin-1; IL-6, interleukin-6; MMP9, matrix metalloproteinase 9; YAP, yes-associated protein; p-YAP, phosphorylated YAP.

VECs. Recent studies have revealed that Ang II increases EC YAP phosphorylation (23), and that angiotensin-converting enzyme 2 activation attenuates pulmonary vascular remodeling via the induction of pulmonary arterial cell apoptosis by upregulating YAP phosphorylation (24). The present results were consistent with these recent studies because they indicated that Ang II increased YAP phosphorylation and regulated VEC activity.

The vascular endothelium has wide-ranging functions that maintain internal homeostasis. VECs form a monolayer that serves as a barrier and separates the basal laminae from the circulation, preventing thrombosis or hemorrhage (6). Additionally, VECs produce and release vasoactive factors, both relaxative and constrictive, to regulate vascular tone and blood pressure (25). Elevation of Ang II is associated with numerous cardiovascular diseases, including pathological hypertrophy, atherosclerosis, aortic aneurysm, heart failure and hypertension (26). In the current study, YAP was revealed to influence the proliferation and inflammation of Ang II in VECs, which should be accounted for in future investigations regarding endothelial dysfunction.
It was revealed that Ang II promoted YAP phosphorylation in VECs via binding to AT1R, leading to the downregulation of VEC proliferation and VEC-mediated inflammation. Therefore, it may be the case that Ang II damages ECs by regulating YAP. An investigation into the hypothesis that enhancing YAP dephosphorylation in VECs will help prevent vascular intimal injury in vivo, should be conducted.

$\mathrm{AD}$ is one of the most severe cardiovascular diseases (27), but its pathogenesis remains to be elucidated. Medical treatment for $\mathrm{AD}$ primarily consists of controlling blood pressure, but there are no clear guidelines for clinical drug use (4). Therefore, investigation of $\mathrm{AD}$ pathogenesis and the generation of clinical recommendations may confer great benefit on patients. In the present study, it was discovered that ARB binds to AT1R on the surface of VECs, thereby regulating downstream YAP and reducing the proinflammatory effects of VECs. The present results suggest that treatment with ARB may reduce VEC inflammation and increase their proliferation. Notably, the effect of Ang II on endothelial cells may contribute to $\mathrm{AD}$ pathogenesis because the primary difference between $\mathrm{AD}$ and aortic aneurysm is the formation 
A

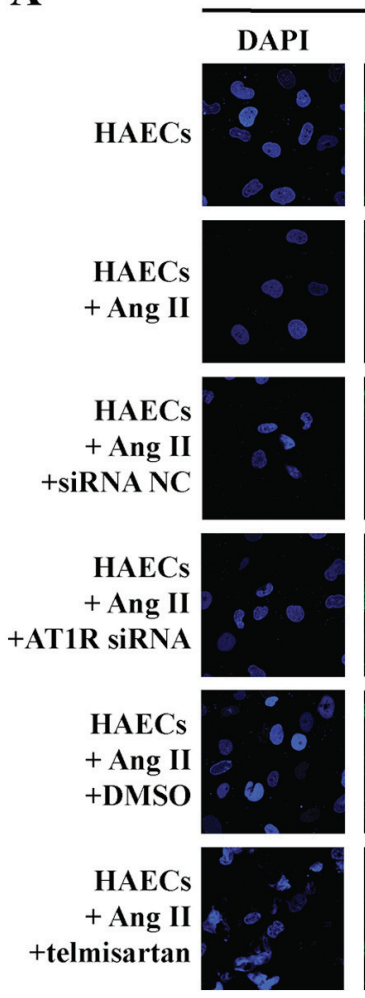

$0 \mathrm{~h}$
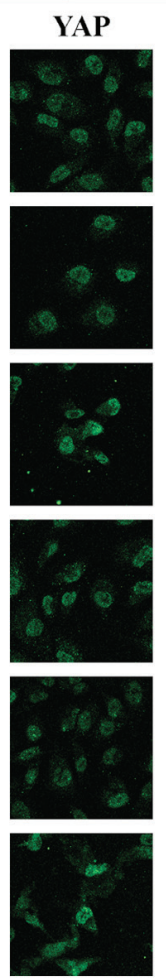

Merge
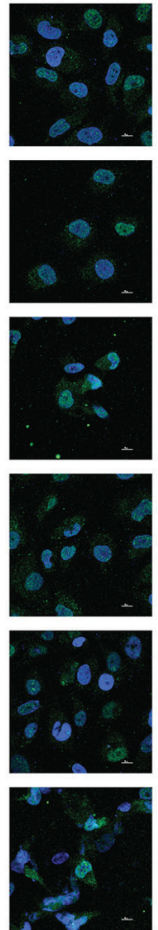
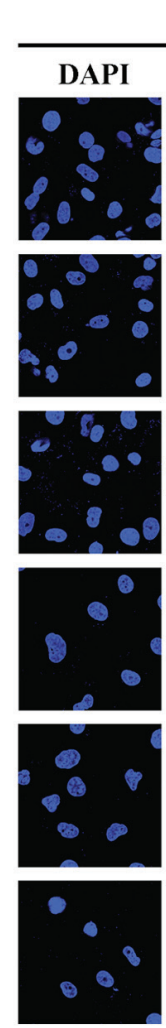

$96 \mathrm{~h}$
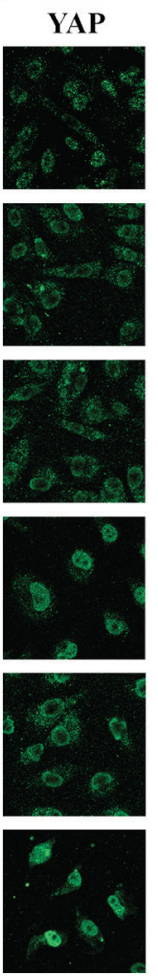

B

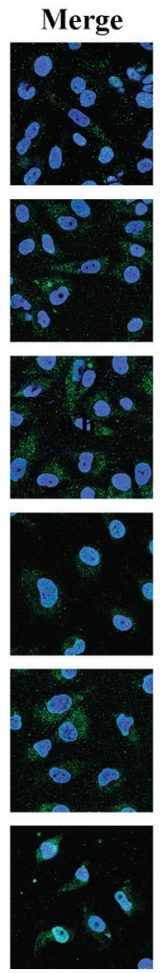

C
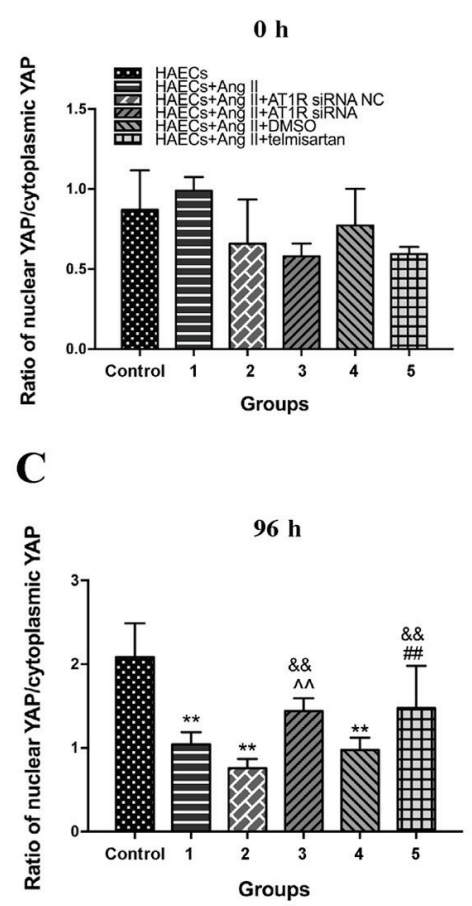

Figure 4. Ang II prevents YAP from entering the nucleus by binding to AT1R. Telmisartan and AT1R siRNA attenuated these effects and promoted YAP into the nucleus. Grouping: Control group, HAECs only; group 1, HAECs with Ang II (1 $\mu \mathrm{M})$ treatment; group 2, HAECs with Ang II (1 $\mu \mathrm{M})$ and siRNA NC transfection; group 3, HAECs with Ang II (1 $\mu \mathrm{M})$ and AT1R siRNA transfection; group 4, HAECs with Ang II (1 $\mu$ M) and $1 \mu 1$ DMSO; and group 5 , HAECs with Ang II $(1 \mu \mathrm{M})$ and the ARB $1 \mu \mathrm{l}(20 \mathrm{mM})$ telmisartan treatment. (A) Fluorescence staining of YAP (green) intracellular location of different groups. Nuclei were labeled with DAPI (blue), and after $96 \mathrm{~h}$ of incubation, YAP was concentrated in the cytoplasm under the influence of Ang II. Telmisartan treatment and AT1R siRNA transfection caused a large amount of YAP to enter the nucleus. (B) Ratio between the amount of YAP in the nucleus and the total amount of intracellular YAP at $0 \mathrm{~h}$. (C) Ratio between the amount of YAP in the nucleus and the total amount of intracellular YAP at $96 \mathrm{~h}$. Mean $\pm \mathrm{SD}$. ${ }^{* *} \mathrm{P}<0.05$ vs. the group control; ${ }^{\wedge} \mathrm{P}<0.05$ vs. the group $1 ;{ }^{\# \#} \mathrm{P}<0.05$ vs. the group 4 ; ${ }^{\&} \& \mathrm{P}<0.05$ vs. the group 2 . siRNA, small interfering RNA; NC, negative control; AT1R, angiotensin type 1 receptor; Ang II, angiotensin II; HAEC, human aortic endothelial cell; YAP, yes-associated protein; p-YAP, phosphorylated YAP.

of intimal tears. Multiple studies have combined AD and aortic aneurysm when studying disease mechanism $(28,29)$; however, the results of the present study indicate that this may be inappropriate. Perhaps the two diseases share certain pathological mechanisms, but understanding the mechanism underlying intimal ruptures is key to elucidating the pathogenesis of AD. Therefore, in the present study, certain preliminary explorations into the effect of Ang II on endometrial cells were conducted; in order provide guidance for future animal experiments on the formation of intimal tears in patients with AD.

The present study had some limitations. First, all experiments were conducted in vitro, so in vivo studies are required to further validate the findings. Second, the effects of YAP overexpression and inhibition on VEC proliferation and inflammation were not investigated due to technical reasons and financial constraints. Further experiments to verify the regulatory role of YAP in VEC proliferation and inflammation should be conducted.

Overall, the present study revealed the integrated roles of AT1R and YAP in regulating VEC proliferation and inflammation in vascular intimal injury and provided an important reference for future research concerning AD formation. The role of YAP in VECs is an important focus for further study, and may represent a promising target for future pharmacological intervention in vascular intimal injury.

\section{Acknowledgements}

The authors would like to thank Dr Sarah Williams for editing the English text for a draft of this manuscript.

\section{Funding}

No funding was received.

\section{Availability of data and materials}

The datasets used and/or analyzed during the current study are available from the corresponding author on reasonable request.

\section{Authors' contributions}

XW, HZ and WG conceived and designed the study. YG, JL and DR analyzed and interpreted the data. XW drafted the manuscript. WG critically revised the manuscript. XW, LC, YH, GS and SJ performed cell culture and experimental tests. All authors read and approved the final version of the manuscript.

\section{Ethics approval and consent to participate}

Not applicable. 


\section{Patient consent for publication}

Not applicable.

\section{Competing interests}

The authors declare that they have no competing interests.

\section{References}

1. Hiratzka LF, Bakris GL, Beckman JA, Bersin RM, Carr VF, Casey DE Jr, Eagle KA, Hermann LK, Isselbacher EM, Kazerooni EA, et al: 20102010 ACCF/AHA/ AATS/ACR/ASA/SCA/SCAI/SIR/STS/SVM guidelines for the diagnosis and management of patients with thoracic aortic disease. A report of the American college of cardiology foundation/American heart association task force on practice guidelines, American association for thoracic surgery, American college of radiology, American stroke association, society of cardiovascular anesthesiologists, society for cardiovascular angiography and interventions, society of interventional radiology, society of thoracic surgeons, and society for vascular medicine. J Am Coll Cardiol 55: e27-e129, 2010.

2. Kochanek KD, Xu J, Murphy SL, Miniño AM and Kung HC: Deaths: Final data for 2009. Natl Vital Stat Rep 60 1-116, 2011.

3. Olsson C, Thelin S, Ståhle E, Ekbom A and Granath F: Thoracic aortic aneurysm and dissection: Increasing prevalence and improved outcomes reported in a nationwide population-based study of more than 14,000 cases from 1987 to 2002 Circulation 114: 2611-2618, 2006.

4. Erbel R, Aboyans V, Boileau C, Bossone E, Bartolomeo RD, Eggebrecht H, Evangelista A, Falk V, Frank H, Gaemperli O, et al: 2014 ESC guidelines on the diagnosis and treatment of aortic diseases: Document covering acute and chronic aortic diseases of the thoracic and abdominal aorta of the adult. The task force for the diagnosis and treatment of aortic diseases of the European society of cardiology (ESC). Eur Heart J 35: 2873-926, 2014.

5. Chen K, Varon J, Wenker OC, Judge DK, Fromm RE Jr and Sternbach G: Acute thoracic aortic dissection: The basics. J Emerg Med 15: 859-867, 1997.

6. Davignon J and Ganz P: Role of endothelial dysfunction in atherosclerosis. Circulation 109 (Suppl 1): III27-III32, 2004

7. Han L, Dai L, Zhao YF, Li HY, Liu O, Lan F, Jiang WJ and Zhang HJ: CD40L promotes development of acute aortic dissection via induction of inflammation and impairment of endothelial cell function. Aging (Albany NY) 10: 371-385, 2018.

8. Schlüter KD and Wenzel S: Angiotensin II: A hormone involved in and contributing to pro-hypertrophic cardiac networks and target of anti-hypertrophic cross-talks. Pharmacol Ther 119: 311-325, 2008.

9. Dimmeler S, Rippmann V, Weiland U, Haendeler J and Zeiher AM: Angiotensin II induces apoptosis of human endothelial cells. Protective effect of nitric oxide. Circ Res 81: 970-976, 1997.

10. Daugherty A, Manning MW and Cassis LA: Angiotensin II promotes atherosclerotic lesions and aneurysms in apolipoprotein E-deficient mice. J Clin Invest 105: 1605-1612, 2000.

11. Sata M and Fukuda D: Crucial role of renin-angiotensin system in the pathogenesis of atherosclerosis. J Med Invest 57: 12-25, 2010.

12. Valis K, Prochazka L, Boura E, Chladova J, Obsil T, Rohlena J, Truksa J, Dong LF, Ralph SJ and Neuzil J: Hippo/Mst1 stimulates transcription of the proapoptotic mediator NOXA in a FoxO1-dependent manner. Cancer Res 71: 946-954, 2011.
13. Ren A, Yan G, You B and Sun J: Down-regulation of mammalian sterile 20 -like kinase 1 by heat shock protein 70 mediates cisplatin resistance in prostate cancer cells. Cancer Res 68: 2266-2274, 2008

14. Hu J, Liu T, Zhang Z, Xu Y and Zhu F: Oxidized low-density lipoprotein promotes vascular endothelial cell dysfunction by stimulating miR-496 expression and inhibiting the Hippo pathway effector YAP. Cell Biol Int 43: 528-538, 2019.

15. Feng X, Liu P, Zhou X, Li MT, Li FL, Wang Z, Meng Z, Sun YP, Yu Y, Xiong Y, et al: Thromboxane A2 activates YAP/TAZ protein to induce vascular smooth muscle cell proliferation and migration. J Biol Chem 291: 18947-18958, 2016.

16. Wennmann DO, Vollenbröker B, Eckart AK, Bonse J, Erdmann F, Wolters DA, Schenk LK, Schulze U, Kremerskothen J, Weide T and Pavenstädt H: The Hippo pathway is controlled by Angiotensin II signaling and its reactivation induces apoptosis in podocytes. Cell Death Dis 5: e1519, 2014.

17. Livak KJ and Schmittgen TD: Analysis of relative gene expression data using real-time quantitative PCR and the 2(-Delta Delta C(T)) method. Methods 25: 402-408, 2001.

18. Pacurari M, Kafoury R, Tchounwou PB and Ndebele K: The Renin-Angiotensin-aldosterone system in vascular inflammation and remodeling. Int J Inflam 2014: 689360, 2014.

19. Nurden AT: Platelets, inflammation and tissue regeneration. Thromb Haemost 105 (Suppl 1): S13-S33, 2011.

20. Wen D, Zhou XL, Li JJ and Hui RT: Biomarkers in aortic dissection. Clin Chim Acta 412: 688-695, 2011.

21. Tsai MH, Wu CH, Lin WN, Cheng CY, Chuang CC, Chang KT, Jiang RS, Hsu JF and Lee IT: Infection with Staphylococcus aureus elicits COX-2/PGE2/IL-6/MMP-9-dependent aorta inflammation via the inhibition of intracellular ROS production. Biomed Pharmacother 107: 889-900, 2018.

22. Yu FX, Zhao B, Panupinthu N, Jewell JL, Lian I, Wang LH, Zhao J, Yuan H, Tumaneng K, Li H, et al: Regulation of the Hippo-YAP pathway by G-protein-coupled receptor signaling. Cell 150: 780-791, 2012.

23. Fu Y, Sun S, Sun H, Peng J, Ma X, Bao L, Ji R, Luo C, Gao C, Zhang $X$ and Jin Y: Scutellarin exerts protective effects against atherosclerosis in rats by regulating the Hippo-FOXO3A and PI3K/AKT signaling pathways. J Cell Physiol 234: 18131-18145, 2019.

24. Yan D, Li G, Zhang Y and Liu Y: Angiotensin-converting enzyme 2 activation suppresses pulmonary vascular remodeling by inducing apoptosis through the Hippo signaling pathway in rats with pulmonary arterial hypertension. Clin Exp Hypertens 41: 589-598, 2019.

25. Shao J, Weng X, Zhuo L, Yu L, Li Z, Shen K, Xu W, Fang M and $\mathrm{Xu}$ Y: Angiotensin II induced CSF1 transcription is mediated by a crosstalk between different epigenetic factors in vascular endothelial cells. Biochim Biophys Acta Gene Regul Mech 1862: 1-11, 2019.

26. Saito $\mathrm{Y}$ and Berk BC: Transactivation: A novel signaling pathway from angiotensin II to tyrosine kinase receptors. J Mol Cell Cardiol 33: 3-7, 2001

27. Nienaber CA, Rousseau H, Eggebrecht H, Kische S, Fattori R, Rehders TC, Kundt G, Scheinert D, Czerny M, Kleinfeldt T, et al: Randomized comparison of strategies for type B aortic dissection: The iNvestigation of STEnt grafts in aortic dissection INSTEAD) trial. Circulation 120: 2519-2528, 2009.

28. Wang S, Liu Y, Zhao G, He L, Fu Y, Yu C, Wang Z, Zhao T, Cao F, Gao Y, et al: Postnatal deficiency of ADAMTS1 ameliorates thoracic aortic aneurysm and dissection in mice. Exp Physiol 103: 1717-1731, 2018

29. Wang Y, Yin P, Chen YH, Yu YS, Ye WX, Huang HY, Ji ZC and Shen ZY: A functional variant of SMAD4 enhances macrophage recruitment and inflammatory response via TGF- $\beta$ signal activation in Thoracic aortic aneurysm and dissection. Aging (Albany NY) 10: 3683-3701, 2018 